\title{
27 \\ EDI in Australian International Trade and Transport
}

\author{
J. Cameron \\ Info.T.EC Solutions Pty Ltd \\ 705/73 Victoria Street, Potts Point, NSW 2011, Australia \\ Phone:+61 23269430 Fax:+61 22181508 Email:cameronj@acslink.aone.net.au
}

\begin{abstract}
EDI has been a "promising" tool for about twenty years. The question that must be asked is why, if it was so "promising", has it taken so long for EDI to be adopted as a standard tool in international trade and transport. This paper examines EDI use in the Australian international trade and transport community and identifies the main inhibitors and constraints. Strategies used by key organisations to encourage adoption of EDI for trade facilitation are described and their success is assessed. Implications for government and international trade and transport are identified. The paper concludes that even when the main macro-level inhibitors and constraints are removed, it is the micro-level economic and market concerns of individual organisations throughout the trading chain that determine successful adoption of EDI.
\end{abstract}

\section{Keywords}

EDI, Electronic Commerce, Trade, Transport, Strategy, Implementation, Inhibitor, Constraint

\section{$1 \quad$ INTRODUCTION}

International trade and transport requires exchange of data and documents among organisations of vastly different sizes and functions located in diverse countries and cultures in different stages of economic, technical and legal development. EDI, defined in this paper as the use of standard, structured messages for computer to computer communication via Value Added Networks (VANs) or via dedicated lines, is considered as a means of facilitating the flow of data and documents and simplifying international trade and transport. Although the extent of EDI usage throughout the world is not known, it was estimated that in 1995 about 50 countries were "in transition from paper-based trade information processing systems to EDI trade facilitation systems" (p17-19 Schware and Kimberley, 1995a). Of the estimated 131,700 
EDI users world-wide, only a small percentage (probably less than 10\%) was involved in trade facilitation. EDI is not a standard tool in international trade and transport.

"EDI in Australian international trade and transportation comprises great and even worldleading success in relation to regulatory transactions, coupled with very limited progress in relation to commercial and operational data flows."(p133 Clarke, 1994). Clarke defines:

- Regulatory data-flows as "..flows to and from customs, quarantine and safety authorities"

- Commercial data flows as messages like "..bookings, forwarding instructions, manifests, bills of lading and waybills"

- Operational data flows as "..those associated with cargo-handling, container tracking and vehicle scheduling".

In order to ascertain the reasons for the successful use of EDI for regulatory messages and the lack of success in implementing commercial and operational EDI messages, the following case studies are described:

- Australian Customs Service Electronics Initiatives which involved regulatory messages

- Tradegate ELECTRA projects that involved commercial and operational messages.

This paper examines the Australian macro-level infrastructure for EDI, estimates usage of EDI in trade and transport, identifies main inhibitors and constraints, and assesses the success of strategies aimed at increasing the use of EDI for trade facilitation. Experience gained in implementing Customs Electronics Initiatives directly influenced strategies used in the ELECTRA projects. Despite significant progress in achieving initial critical mass resulting from successful implementation of Customs EDI systems and the removal of many inhibitors and constraints, the paper concludes that there is no guarantee that EDI will be adopted as a standard tool in international trade and transport.

\section{Government and Industry Support for EDI}

In 1986 the Commonwealth Government established an inter-State Commission to improve information flows and communications within the trade and transport industries. The National Communications Working Party on Cargo Movements, set up by the Waterfront Industry Committee, recommended the establishment of a national communications network for EDI in 1988. Tradegate Australia Limited was consequently set up in August 1989. Founding members of Tradegate were the industry bodies representing road and rail carriers, shipping lines, freight forwarders, customs brokers and importers, port authorities, Customs, Austrade (representing exporters) and Qantas (representing airlines). Tradegate's charter was to "improve the efficiency and speed of moving goods through the trading chain by the widespread introduction to the Australian trading community of a comprehensive range of paperless trading services" (Tradegate marketing brochure 1993). In 1989 Tradegate was awarded a contract by Customs to supply electronic network services to Customs' users until the end of 1996. Tradegate entered an agreement with AT\&T's predecessors that provides a percentage royalty (related to the kilocharacters transmitted by its members) and discounts to all members based on total volume. Tradegate lowered average service costs for all members by $20-30 \%$ below the normal prices charged by VANs and obtained funds for its activities. 


\section{Legal, Regulatory and Commercial Considerations}

Legal issues and considerations no longer "present any major impediments to EDI implementations" although "much of the law needs revision to be consistent with EDI" (p1, p4 Schware and Kimberley, 1995b). Trading partner agreements are used to deal with most legal and contractual issues within Australia and with established trading partners in many other countries. The use of EDI is simplified in trade between countries and organisations utilising similar legal and commercial frameworks and business practices. Factors like the use of the Bill of Lading (a negotiable instrument and document of title), regulatory requirements and banking practice related to ownership of goods and payments, do limit the use of electronic trading in some markets and for some commodities. However, EDI messaging between New Zealand and Australia is increasing significantly as trade practices are changed at regulatory and commercial levels. Eg. Sea waybills replace Bills of Lading for $70 \%$ of consignments in Trans-Tasman trade.

\section{Technical Infrastructure}

Australia has a mature IT industry and a modern telecommunications infrastructure. VAN services are offered by AT\&T, Telstra, GEIS, IBM and SITA, there are numerous Internet Service Providers and on-line access to port operations information, vessel movements and schedules, terminal operations and freight availability. File translation and file, fax and email conversion facilities are available to allow exchange of structured messages among organisations that are EDI-enabled and standards compliant and those that are not.

\section{Technical Skills}

Australia has a mature software and hardware vendor infrastructure and a skilled IT profession. Australians are rapid adopters of IT. Most large organisations in the trade and transport industry are now fully computerised. However, many small and some medium-sized businesses are not sophisticated in their use of IT.

\section{Standards and Internationally Harmonised EDI Message Guidelines}

Associations working for the implementation of standards related to EDI include Electronic Commerce Australia, European Article Numbering Association (EAN), Standards Australia and Tradegate. Regulatory message standards are determined by the appropriate Australian government organisation. Customs has adopted some of the relevant UN-EDIFACT guidelines but does use proprietary formats for messages related to internal reporting requirements. Discussions between New Zealand and Australian Customs have standardised some international regulatory messages.

Australia is well represented in the UN-EDIFACT transport message development process. An Australian from Tradegate co-chairs the JRT4 Working Party that reports to UN/ECE WP.4/GE.1, the group that ratifies message standards for international use. Business needs of Australian and New Zealand users, identified as a part of the Tradegate ELECTRA projects in 1995, were incorporated into internationally harmonised message implementation guidelines in the D94B Directory format for the International Forwarding and Transport Message set (IFTM). Countries using harmonised guidelines are able to exchange commercial trade and transport messages relatively easily. Operational messages related to Dangerous Goods, maritime messages like status reports, terminal performance and stowage, etc. are not yet 
harmonised. Container Handling Message guidelines will be available in D95A Directory format. The Bay Plan message (BAPLIE) was harmonised in 1994. Over 1,000 implementations have been reported internationally. The International Airline Transport Association (IATA) develops operational messages for airline use. CargolMP (Cargo Interchange Message Procedures) is the standard currently used.

\section{Summary}

The macro-level environment in Australia provides the preconditions necessary for successful implementation of EDI as a standard tool for international trade and transport. In addition, 3 of Australia's 7 major trading partners (USA, New Zealand, United Kingdom) operate in similar legal, regulatory and commercial environments, speak the same language and have the same cultural roots and Singapore is a sophisticated user of EDI for trade facilitation.

The Australian trade and transport industry is estimated to comprise about 20 government bodies, 38 port authorities, 80 shipping lines and agents, 200 freight forwarders, 450 customs brokers, 800 road carriers, 6 railways, 20 container terminals and stevedores, 150 depots (unpack and storage), 40 container parks, 30 airlines, 20 banks, 10 insurance companies, 3,000 exporters and more than 30,000 importers (industry sources). Most trading chains therefore involve small and medium sized businesses.

In January 1995 about 9 million trade and transport EDI messages were exchanged annually. The annual volume expected for 1996 is 11 million messages. Regulatory messages comprise about $90 \%$ of all EDI messages and the remaining $10 \%$ are commercial and operational. Tradegate (via outsourcing arrangements with VANs) handles about $95 \%$ of regulatory messages. About $50 \%$ of commercial and operational messages are transmitted via Tradegate/ AT\&T, possibly $25 \%$ utilise other VANs and $25 \%$ are carried via direct connections.

In mid 1994 over 2,200 member sites were connected via Tradegate outsourced networks (Tradegate Corporate Brochure, 1994) and about 50\% of members used EDI services (Clarke 1994). In February 1996 Tradegate had 2,400 sites connected. From July 1994 the total EDI kilocharacters transmitted by Tradegate sites rose steadily from about 300 million characters per month to over 400 million characters per month in February 1996. Most are regulatory messages transmitted to and from Customs. Average message size is about 400 kilocharacters.

The percentage of commercial transport messages transmitted has remained constant at less than $1 \%$ of total messages and kilocharacters. Volume rose significantly in July 1995 (an increase of 3.5 million characters from June to July), peaking in August and October at over 4 million characters and 8,000 messages a month when the Tradegate ELECTRA projects were piloted. This volume remained relatively steady until February 1996. Available usage statistics confirm, that as in 1994, the use of EDI for commercial and operational messages is very limited in 1996 despite the high volume of regulatory messages. The potential for increased use of EDI for commercial messages is enormous. Tradegate estimates the penetration rate for EDI in March 1996 at about 7-8\% of potential. 
Customs is the most important "hub" organisation for international trade and transport. Customs aims to protect the community and to facilitate trade and transport. Computerisation is used to achieve both aims. The INSPECT batch system, for collecting and checking entry information supplied by importers and agents, was implemented in 1972 followed in 1974 by the SEARCH system that gave Customs officers access to this data.

By the mid-1980s the increased complexity of Customs' role (from collection of duty and prevention of smuggling into tasks like compilation of external trade statistics), combined with an increased volume of international trade, meant that Customs was finding it difficult to accurately and consistently compare data in import and export documents and to assess risk manually. Information flows and communication within the trade and transport industries and cargo processing speed (particularly air freight) needed improvement at a time when Customs was required to achieve internal efficiencies and to reduce staff. There was pressure to balance control with facilitation and simplification of procedures to reduce costs to trade and transport. (Australian Customs Service, 1993). The Electronic Initiatives Charter of the Australian Customs Service stated that "custom's ultimate aim is to have all its cargo systems automated by 1995" (Australian Customs Service newsheet, November 1989). Suitable software, hardware and communications technology was available and many key participants in the trade and transport industry were using computers. EDI was selected in preference to direct access or on-line entry because there was "a strong industry as well as technical view that EDI would become an integral part of industry management and business methods"; EDI met cost and efficiency criteria and government strategy supported the choice of EDI (p20 Tradegate, 1995). The decision was reinforced by the acceptance of UN/EDIFACT as the international standard for EDI and by adoption of EDI for customs operations around the world by Customs Co-operation Council members in 1990.

\subsection{Customs Systems and Usage}

Systems comprising the Electronics Initiative Strategy, their functions and usage are:

1. EXIT 1 implemented in 1988 allows exporters to submit electronic requests for clearance and issues Export Clearance Numbers (ECNs). The use of this EDI system was made mandatory in 1991 because of poor take-up. By 1994 about $97 \%$ of ECNs were applied for and issued via EXIT 1 (p126 Clarke, 1994).

2. EXIT 2 an EDI system implemented in 1991 allows manifests to be cleared electronically. By $199489 \%$ of Master Air Waybills were submitted electronically by airlines, but very few freight forwarders lodged House Waybills and only about $20 \%$ of sea cargo was reported electronically (p126 Clarke, 1994). Full implementation of risk assessment and profiling facilities for exports is being implemented in 1996.

3. COMPILE a direct on-line system, implemented in 1976 and updated in 1986 to replaced the INSPECT and SEARCH systems, enables importers and customs brokers to create and lodge entries using in-house terminals. It establishes intention to import. Interim EDIFICE, an on-line PC software package, was implemented in 1990. By $199598 \%$ of all import entries (2.1 million entries per year) were input by 620 users (Tradegate statistics).

4. EDIFICE a full EDI system based on UN/EDIFACT standards was implemented 1995-6. 
5. Electronic Funds Transfer, connected to COMPILE in 1990, automates payment of import duty. By $199597.5 \%$ of import entries were paid through EFT. (Tradegate)

6. Tariff and Precedents Information Network (TAPIN) provides details of tariff items for Customs Brokers. It is a direct entry on-line system implemented in 1991.

7. Air Cargo Automation (ACA), an EDI system implemented progressively from 1990 to 1992, automates air cargo control, increases the speed of cargo processing and improves the efficiency of risk identification.

8. Sea Cargo Automation (SCA) automates control of containerised sea cargo and improves efficiency. Implemented progressively from 1993 to 1995 , SCA is the maritime equivalent of ACA. It establishes the fact that importation has occurred.

9. EXDOC implemented in 1992 integrates Customs with quarantine entries so that consignments of food products can be cleared.

\subsection{Lessons Leant}

Key factors in increasing the take up of ACA were (Ch.3 p14 Cameron, 1993):

- Competitive advantage achieved by larger companies (especially integrated carriers handling large volumes of cargo, door to door deliveries and express freight) due to:

- their technical knowledge of and operational experience with EDI

- faster customs clearance (cargo released within 15 minutes compared with the 4-6 hours delay prior to ACA implementation).

- use of electronic data for management purposes.

- Market pressures, especially the need to respond to competition.

- Availability of suitable software.

- Increased benefits of computerisation and automated data entry offsetting some EDI costs.

- Marketing and education.

- Customs gradually extending the time to process manual Air Waybills.

Take-up rate of ACA among freight forwarders and handlers was lower than expected. ACA imposed additional costs without immediate commercial benefits when:

1. Cost/benefits of automation were not achieved Eg. Rekeying to stand-alone PCs continued

2. Business issues and needs were not adequately addressed

3. Industry culture was not conducive to automation

4. Knowledge of IT and EDI was not available at reasonable cost

5. Suitable PC software was not available

6. System complexity, combined with high turnover of staff, led to high error rates.

When the Sea Cargo Automation System (SCA) was implemented between March 1993 and 1995, Customs' strategies were changed as follows (pp14-5 \& p22 Tradegate, 1995):

1. To address issues related to cost

Although commercial practice normally means that the sender of electronic messages pays the transmission cost, users of Customs systems were required to pay both the costs of data sent to and received from the department. In order to reduce costs to users, from 1 July 1995 Customs paid the costs of messages sent by the department to users. Internal costs of EDI use and implementation remained the concern of each enterprise. 
2. To address business issues

Industry organisations and Customs worked in closer partnership to develop and implement SCA. Industry was more willing to participate after the experience of ACA. "Best practice workshops" were held to help Customs resolve design, development and implementation issues and for users to assist each other with business issues. Implementation Committees with a local focus, were established at each port to deal with matters specific to that locality.

3. To address lack of knowledge of EDI

Customs provided "teach-in" training, Tradegate provided simulation exercises in all ports and software suppliers provided hands-on training and support. IT literacy had increased.

4. To address system complexity

More effective involvement of users in the design and development resulted in a less complex user interface. User testing was introduced as a prerequisite to live connection to SCA.

Problems related to the provision of PC software by suppliers still occurred. The cost/benefit of using EDI for smaller enterprises remained questionable. Consultation with users, although improved, was not as effective as required. For future Customs implementations, strategies will be further refined to include: (p21, 28-29 Tradegate, 1995)

- Joint working relationships between industry and Customs, possibly allowing direct consultation with users to overcome difficulties in ensuring effective communication among industry associations and the members. Formal memoranda of understanding with associations regarding their roles and responsibilities may be negotiated.

- Industry representatives seconded to work full-time with Customs' development teams.

- Intra-industry process re-engineering to ensure the full benefits of automation and EDI are achieved (eg. Banks to allow automatic lodgement of trade related documents, etc.).

- Use of standard UN-EDIFACT messages by Customs where possible.

- Use of existing commercial documentation as the basis of data collected by Customs.

\subsection{Summary}

Customs was successful in implementing it Electronic Initiatives Strategies due partly to its position as regulator, but also because it was prepared to revise its strategies to address some of the main inhibitors and constraints affecting the take-up of EDI by private sector enterprises. The support of Tradegate as community facilitator and electronic network provider was very important. Nevertheless, industry members knew that Customs had the power to mandate the use of EDI, to delay the manual processing of freight for periods that were unacceptable to clients and/or to impose financial and other penalties on non-compliant enterprises. Customs' ability to mandate provided an initial critical mass of EDI users.

Despite suitable macro level infrastructure, and successful implementation of EDI for regulatory messages that provided a critical mass of EDI users in the parts of the trading chain interacting with Customs, commercial and operational messaging was not widely used in Australia at the beginning of 1996. The major clients of the international trade and transport chain, exporters and importers, were not demanding the use of EDI. Most of their 
international trading partners were not EDI-enabled and they could not use EDI through the Australian trading chain. The reasons for the lack of use of EDI relate to factors at the enterprise level.

\subsection{Micro- Level - Inhibitors and Constraints Within Enterprises}

The reluctance of private sector enterprises in the Australian international trade and transport sectors to use EDI in 1994 related to the following factors:

\section{Lack of knowledge of EDI and IT expertise}

Many organisations involved in the trade and transport industry (eg. road carriers) are not required to exchange regulatory data with Customs. For these enterprises EDI was a new concept and many small companies were not computerised. In December 1994 a survey of 48 Tradegate members invited to be involved in the EXTEDI project found that only 12 had used EDI ( 9 for commercial messages; 7 were linked to Customs and 4 used EDI for operational messages) (pp42-6 Cameron and Jeacle, 1995). Four respondents had been using EDI with Customs for 5 years and were also using EDI for other messages.

\section{Lack of trading partners}

Unlike regulatory messages, which are normally transmitted between one user and a regulator, commercial messages flow through a trading chain. If one link is not EDI-enabled the flow is hindered and benefits are significantly reduced. Operational messages generally pass between two parties that are often large organisations like shipping lines and container terminals.

\section{Cost/Benefits}

Establishment costs of EDI are comparatively high for small organisations with low message volume. Even for organisations already sending EDI regulatory messages, costs of acquiring additional software for commercial or operational messages may be considered high. When assessing costs of manual processes many businesses do not record all cost (eg time to deliver documents). This distorts the comparative costs of EDI. And if EDI is not integrated with other business applications, the full benefits are not realised. Dual manual and automated procedures are expensive to maintain. Business processes and practices need to be reengineered. Industry and organisational culture must be changed. The obvious benefits of EDI messages accrue mainly to the receiver because they do not have to key data (p130, Clarke, 1994). Although EDI provides additional management data and enables new services to be provided to clients, these advantages may not be recognised. Without client demand for EDI, there are few benefits related to retained business.

4. Management of implementation among trading partners

The competitive nature of enterprises in the transport industry and competition within parts of the trading chain, combined with concerns about the impact of EDI on future viability of some organisations (eg. small freight forwarders) means that collaboration for implementing new systems or procedures does not occur naturally. The few attempts at implementing EDI within trading chains were undertaken to achieve competitive advantage. Action from a community facilitator and co-operation from industry associations was required to co-ordinate and achieve EDI implementation for trade facilitation.

\section{Lack of business motivation}

If not pushed by major clients there may be little incentive for enterprises to implement commercial and operational EDI messaging unless there are internal cost/benefits to be 
achieved. Larger and/or computerised companies receive the most benefit from EDI. Eg. Large shipping lines/agents decided to undertake initial data entry from telephone bookings from smaller clients in order to avoid having to maintaining dual manual and EDI procedures.

6. Regulatory issues and commercial practice

Some organisations were concerned about message security, legal matters related to the use of EDI for international trade, difficulties in negotiating trading partner agreements and legal contracts, and problems with banks and negotiable instruments (p44 Cameron and Jeacle, 1995). In the maritime trade there was a reluctance to use a waybill rather than the Bill of Lading (a signed paper document), even if it was permitted by regulation and ownership of goods was not to be transferred during shipment. Five hundred years of tradition is difficult to change! Waybills are used exclusively and successfully for international air freight.

7. Message standards

UN-EDIFACT standards for operational messages were not complete. Many messages used proprietary formats. Eg. On-line vehicle booking systems for trucks loading/unloading from wharves. Australian Chamber of Shipping (ACOS) operational messages related to container movements were used by shipping lines and container terminals. Commercial UN-EDIFACT messages were used in maritime trade, but the D91.2 Directory used was not upwardly compatible. And not all software adhered to the same implementation guidelines. The use of multiple standards hindered the potential for connecting with other industry groups like the retailers and manufacturers.

8. EDI-enabled software

Some of the in-house and vendor supplied software packages available were incompatible. This made EDI implementation complicated, frustrating and expensive.

9. Communications

Interconnections among some VANs and equipment within Australia and overseas were not reliable.

\subsection{Tradegate Strategies}

In 1994, after a successful APEC (Asia Pacific Economic Community) pilot study using EDI for commercial and regulatory messages in Trans-Tasman trade proved the viability of EDI for international trade and transport, Tradegate began the ELECTRA projects proposed by the National Consultative Group on Transport EDI (p135 NCG, 1994). Funding was provided by Tradegate, Commonwealth Department of Transport and Electronic Commerce Australia. Experienced project managers were employed as facilitators. AIREDI focused on commercial EDI messages for exports and imports by air, EDIMI dealt with commercial EDI messages for maritime imports and financial messages related to transport, and EXTEDI's scope originally comprised commercial and operational messages for maritime exports. Project reports are set out in McKittrick, 1995, Assenza and Walsh, 1995 and Cameron and Jeacle, 1995.

To ensure proper consultation within industry occurred, a two-tiered management structure was developed. A steering committee, comprising representatives of industry bodies and sponsors, was responsible for policy and co-ordination. To ensure that business issues were addressed, each project established teams of user organisations active in international trade and transport. Retaining commitment of all members was not possible. Enterprises were diverted by business priorities. Several members became inactive because of changes of staff, 
over commitment of IT personnel and competing IT projects. Some were interested in learning about EDI and contributing to specifying user needs. Eg. EXTEDI user groups comprised 45 enterprises that were actively involved in international trade and transport: 28 were willing to trial messages but due to delays in software availability only 13 were involved in the final tests (Appendices, Cameron and Jeacle, 1995). EDIMI user groups comprised 25 enterprises and 15 were involved in testing (Appendices, Assenza and Walsh, 1995).

Project teams examined business practices and processes, determined data flows and requirements, and developed UN-EDIFACT implementation guidelines for commercial messages. Operational message guidelines were not prepared because EXTEDI users, particularly exporters, determined that booking message guidelines were more economically significant and would lead to greater cost savings for all members of the trading chain. (Eg. South Pacific Tyres Pty Ltd found up to $45 \%$ of data required by waybills is needed for booking cargo). Responsibility for operational message guideline development was transferred to ACOS. Proprietary and existing standards continued to be used.

Initially the project teams spent time understanding the business needs of individual organisations and of parts of the trading chain and in ensuring knowledge of EDI was transferred. Agreement was needed to establish data requirements for different activities and different ways of doing business. Guidelines needed to be flexible to allow for variations in business procedures, requirements associated with specific commodities and means of transport, and changes in commercial practice over time and among trading partners throughout the world. Legal, contractual and commercial issues were addressed. EDIMI worked with the Banking Industry Group to develop EDI guidelines for payment of invoices. EXTEDI and EDIMI project leaders worked closely with the domestic EDI transport project (DOMEDI) to ensure the guidelines were compatible. DOMEDI developed guidelines in both ANSI and UN-EDIFACT format to allow interchange of messages with the retail and manufacturing industries, the main client groups for road and rail carriers.

Software developers were provided with draft implementation guidelines, software was developed and trials were carried out under business conditions. Software vendors were not included in user groups to ensure guideline specifications were business focused and not influenced by technical considerations. Unfortunately some vendors did not develop PC products on time and some did not conform to guideline standards. Maritime and air messages flowed along trading chains within Australia and were tested in Trans-Tasman trade. After testing, some participants adopted the new guidelines and continued live transmissions.

Cost/benefits of EDI were demonstrated. Eg. EDI saved about $\$ 20$ per document due to reduced errors, rekeying, courier costs, delays in delivery and staff time (pp26-7, Cameron and Jeacle, 1995). Improved management information resulted in additional cost savings and improved profit. But the greatest benefit was increased service to clients that gave competitive advantage to early adopters of EDI and offset some cost disadvantages of early innovators. These findings are supported by other case studies (Mc.Cubbrey and Imai, 1994).

After 24 months work by ELECTRA project teams and direct project costs of about $\$ 500,000$, UN-EDIFACT internationally harmonised implementation guidelines for commercial 
messages were published and adopted for use by the New Zealand and Australian trade and transport industries. The availability of harmonised message implementation guidelines will enable new Australian EDI users to exchange EDI messages easily internationally and to avoid the imposition of proprietary standards by large organisations. EDI education and awareness issues were addressed by Tradegate through press releases, progress reports in newsletters and industry publications, and by launching the ELECTRA projects at trade days. Over 600 people registered to attend trade days held in Sydney and Melbourne in March/April, 1996. It is expected that this interest, encouraged by on-going marketing and assistance from Tradegate and existing users, will translate into significantly increased use of EDI throughout the Australian international trade and transport industry.

\subsection{On-Going Inhibitors and Constraints}

Although the strategies used in the ELECTRA projects resolved most of the constraints and inhibitors the following issues remain:

1. Messages standards

Many organisations continue to use non standard messages. The need for translation among formats and maintenance of multiple tables increases complexity and cost. Gateways (eg CargoNet for air freight messages) and translation services are available - at a cost. Additional messages and harmonised guidelines are being prepared, reviewed and refined. Operational message guidelines were completed in April 1996.

2. Rate of take-up - Critical mass

Increased demand for the use of EDI is required from importers and exporters before EDI can become an integral aspect of trading relations. Adoption of EDI for commercial and operational messages is slower than expected but as Australian organisations utilise other forms of electronic commerce, the use of EDI is expected to increase significantly. Links to the Internet and use of email by business is growing exponentially. Community standards simplify exchange of messages across industry boundaries and enhance the viability and convenience of EDI as a way of doing business.

3. Cost of Implementation

Although costs of EDI-enabled software and transmission are reducing, low volume users and small enterprises continue to find EDI implementation expensive. Total cost per message will decrease as EDI and other forms of electronic commerce are used for a variety of purposes.

4. Facilitation and management of implementation

Consultancy services are available, but smaller companies may be discouraged by cost.

5. Software

Interconnectivity of software products continues to be a problem. Not all software packages comply with the guidelines and many enterprises experience difficulties exchanging messages with trading partners using different products. Interfacing EDI software with in-house and/or other business software applications can be costly and time consuming but integration achieves cost/benefits. Supply of suitable software packages remains a problem. For software vendors, commercial benefits are not available until there is a critical mass of users and a viable market. And take-up of EDI is hindered by a lack of suitable software packages!

6. Communications

The interoperability of VANs offering international services needs to be assured. Agreements have been concluded among many large global network operators, but considerable work 
remains. The relative cost of VANs may mean that some organisations will use the Internet and direct connections with trading partners for EDI messaging.

\subsection{Summary}

The ELECTRA projects removed some main inhibitors to EDI use in the trade and transport community. Development of harmonised guidelines for commercial messages removed a major hurdle. Business procedures and practices were reviewed and further streamlining will occur with time and experience. Cost/benefits of EDI for a variety of activities and enterprises were demonstrated. Leadership from EDI-enabled organisations, their willingness to act as "hubs" and to assist trading partners to become EDI-enabled are important precursors to creating market pressure in the industry. Tradegate is now involved in additional APEC projects to establish EDI links at government and enterprise level with major trading partners.

\section{6}

CONCLUSIONS

The implications of the Australian experience for international trade and transport suggest that even when the necessary macro-level infrastructure is available there is no guarantee that EDI will be implemented within industry and used for international trade facilitation. In Australia the achievement of a critical mass of users of EDI for regulatory messages was no guarantee of successful implementation of EDI for commercial and operational messages. Government policy and assistance were less important at the micro-level than economic and business factors like cost/benefit and market forces.

Many studies report that the "hub and spoke" format of EDI take-up, (often related to "upstream EDI") where the client, usually a large organisation, playing a leading role and forming the hub, is the way growth in the use of EDI occurs within industries (p32 Mc.Cubbrey and Imai, 1994 and p175 Lobet-Maris, 1994.) The "hub" is able to impose its standards on the "spokes". If these standards are proprietary, the take-up of EDI with new partners is inhibited. This model occurred in the Customs case study, where the regulator was the hub. However, as the regulator was a monopoly within Australia, the use of non-standard messages for internal regulatory purposes did not hinder the take-up of EDI for commercial and operational messages. The hubs for international trade and transport messages are exporters and importers. In Australia the establishment and use of harmonised UN-EDIFACT message implementation guidelines mean that each hub drives the use of standards and enhances the viability of EDI throughout the trade and transport trading chain.

Another method of EDI diffusion relates to "centralised market exchange" ("down stream EDI") which is implemented for strategic reasons among long-term business partners, often to ensure loyalty (p177 Lobet-Maris, 1994). In Australia this method was used by some EDIenabled shipping lines, freight forwarders, container terminals and a rail operator and their major clients. However, they were not successful in significantly increasing the use of EDI through the trading chain. European studies confirm that "down stream EDI is less successful. Customer and market demands are more powerful (p214 Krcmar et al, 1994). 
Regulatory messages can be implemented by a strong driving organisation. But implementation of commercial and operational EDI messages frequently involves numerous small and medium-sized organisations. The management of collaborative projects is not well understood (Cameron and Clarke, 1996). The process requires collaboration, shared decisionmaking and joint activities like negotiation of contracts, and changing business processes throughout the chain. Most private sector enterprises are competitors and are motivated by economic factors. But successful implementation of EDI and electronic commerce demands co-ordination of "volunteers" and all must achieve a win-win outcome. There is a need for formal facilitation and experienced facilitators, funding, resourcing and commitment.

Internationally, a critical mass of EDI users has not been achieved. In each country government can ensure infrastructure exists and remove impediments related to internal regulatory, legal and commercial practices but they cannot enforce the use of EDI for international trade. Even if governments can control organisations located in their own country, they cannot enforce the use of EDI by enterprises world-wide. Only after extensive negotiation and collaboration among governments and trading partners at the enterprise level will macro-level inhibitors and constraint be removed. But even when appropriate infrastructure and preconditions are established, it will be necessary to address the micro-level economic and market concerns of enterprises throughout the trading chain before EDI will be adopted as a standard tool in international trade and transport.

\section{7} REFERENCES

Assenza, F and Walsh, P (1995) EDIMI - EDI for Maritime Imports: Final Project Report. Tradegate Australia Ltd

Australian Customs Service (1993) Computers in the ACS. Australian Customs Service

Cameron, J (1993) EDI and the Air Cargo Industry. Department of Commerce, Australian National University

Cameron, J and Clarke R (1996) Towards a Theoretical Framework for Collaborative Electronic Commerce Projects Involving Small and Medium-Sized Enterprises. Conference Paper Ninth International EDI-IOS Conference, June 1996

Cameron, J and Jeacle, P (1995) EXTEDI - EDI for Maritime Exports: Final Project Report. Tradegate Australia Ltd

Clarke, R. (1994) EDI in Australian International Trade and Transportation Electronic Commerce Electronic Partnerships (eds. J. Gricar, J. Novak) Proceedings Seventh International EDI-IOS Conference, June 1994

Krcmar, H., Bjorn-Andersen, N., Eistert, T., Grises, J., Jelassi, T., O'Callaghan, R., Pasini, P., Ribbers, P. (1994) EDI in Europe - Empirical Analysis of a Multi-Industry Study Electronic Commerce Electronic Partnerships(eds. J. Gricar, J. Novak) Proceedings Seventh International EDI-IOS Conference, June 1994

Mc.Cubbrey, D. And Imai, T. (1994) EDI/IOS in the U.S. and Japan: Constrasts and Conjectures Electronic Commerce Electronic Partnerships(eds. J. Gricar, J. Novak) Proceedings Seventh International EDI-IOS Conference, June 1994

McKittrick, S. (1995) AIREDI -EDI for Imports \& Exports: Final Project Report. Tradegate Australia Ltd 
NCG (1994) Report on EDI Implementation in the Transport Sector: An Assessment/Stocktake, National Consultative Group of Transport EDI, Department of Transport Canberra

Schware, R. and Kimberley, P. (1995a) Information Technology and National Trade Facilitation: Making the Most of Global Trade. The World Bank, Technical Paper No. 316

Schware, R. and Kimberley, P. (1995b) Information Technology and National Trade Facilitation: Guide to Best Practice. The World Bank, Technical Paper Number 317

Tradegate Australia Ltd (1995) The Australian Customs Service Sea Cargo Automation Systems - A Situation Assessment, Tradegate Australia Ltd

\section{Acknowledgements}

Assistance from Andrew Robertson, Chief Executive, Tradegate Australia Pty Ltd, Graham Bannister, Australian Customs Service and Roger Clarke, Xamax Pty Ltd was very important to this paper. I thank ELECTRA-Project Managers, Tradegate staff and EXTEDI participants.

\section{BIOGRAPHY}

Julie Cameron is an independent consultant with practical experience implementing EDI and electronic commerce in large and small enterprises, combined with case study research and examination of theories related to collaborative projects. Her background includes senior executive roles in private and public sector organisations, including managing information technology, information processing and information and IT policy. Since 1985 she has assessed and managed impacts of IT on organisations and communities. Julie is member of the IFIP Working Groups concerned with computers and work and social accountability as well as a joint Australian representative to Technical Committee 9 - Computers and Society 